\title{
Enhancing Potentiometric Response of Electrochemical Sensor Using Modified Ion-Sensitive Transistor
}

\author{
V. Abhinav ${ }^{\mathrm{a}}$, R. Patkar ${ }^{\mathrm{a}}$, M. Vinchurkar ${ }^{\mathrm{a}}$, T.R.Naik ${ }^{\mathrm{a}}$, and M. S. Baghini ${ }^{\mathrm{a}}$ \\ ${ }^{a}$ Department of Electrical Engineering, Indian Institute of Technology Bombay, \\ Powai, Mumbai 400076, India
}

In this work, we present a novel combination of solid-state ion selective electrode and field effect transistor (FET) integrated on same platform. Thus, creating an extended gate field effect transistor (EGFET). We have built an electrochemical sensing unit comprised of all solid-state electrodes, an $\mathrm{Ag} / \mathrm{AgCl}$ reference electrode and ionophore coated gold electrode. Unlike any of the earlier reports, all the electrodes are integrated with a FET in a single plane of printed circuit board (PCB), hence mitigating the issues of separate wire bonding or external connection between the sensing element and the transducer by using PCB traces. In this work, we demonstrate for the first time, the use of ion sensitive electrode (ISE) in EGFET configuration without degrading the linearity and sensitivity due to the external voltage bias. Potentiometric measurement on our $\mathrm{K}^{+}$ISE shows near Nernstian limit sensitivity (49 $\mathrm{mV} /$ decade), low concentration for the limit of detection $\left(10^{-6} \mathrm{M}\right)$, and linearity over a large range of detection $\left(10^{-4} \mathrm{M}\right.$ to $\left.1 \mathrm{M}\right)$.

\section{Introduction}

High sensitivity, high selectivity, reproducibility and quick response are of prime importance for ion detection in various applications. In this respect, electrochemical detection is one of the best technique as compared to the traditional flame photometry, ion chromatography and surface plasmon resonance [1,2].

In an ion sensitive FET (ISFET), directly exposing the gate oxide of FET to an ionic solution causes the problem of ion penetration into the oxide layer. This issue is reflected in I-V characteristics that show voltage-dependent hysteresis due to ion penetration [3,4]. EGFET shows great potential over traditional ISFETs or ISE. EGFET effectively improves the reliability by separating the sensor and the transducer, associating the two by interconnect. It also provides a wide range of measurement with scope for device miniaturization and ease fabrication [5].

Commercial printed circuit board (PCB) based technology is presented for the low-cost production of electrodes and the same is used for circuit board. PCB technology has been explored by Prodromakis et al. 2011 for $\mathrm{pH}$ monitoring with the sensitivity of $\sim 22 \mathrm{mV} / \mathrm{pH}$ [6]. They have sputtered $\mathrm{H}^{+}$ion sensitive $\mathrm{TiO}_{2}$ of thickness $150 \mathrm{~nm}$ on top of gold plated copper electrodes. Trantidou et al. 2013 have used plasma treatment for deposition of Parylene-C helped it to reduce the drift parameter (2.5-10 mV/hour) but at the cost of sensitivity ( 16 mV/pH) [7]. Further Moschou et al. 2015 have presented high $\mathrm{H}+$ ion sensitive indium tin oxide (ITO) layer with the improved sensitivity (45 mV/pH). 
This paper presents for the first time potassium ion concentration sensing using EGFET with PCB as the substrate [8].

\section{Experiment}

In this work, we demonstrate a simple approach to develop an array of miniaturized two terminal planar solid state electrodes for the EGFET based sensor. Each electrodes consists of an ion selective membrane (ISM) on gold as the working electrode (WE) and solid state silver/silver chloride (Ag/AgCl) as the reference electrode (RE) as shown in figure 1 . Both the electrodes WE and RE are clamped to the metal contacts of the FET. Electro-less nickel immersion gold (ENIG) is used to deposit $200 \mathrm{~nm}$ thick gold electrode as the two terminal device. The working electrode (WE) is coated with ISM cocktail consists of crown ether (18-crown-6), DOS, and PVC dissolved in THF. 18crown-6 (ionophore) has been utilized in ion selective membrane on WE to achieve high selectivity towards $\mathrm{K}^{+}$ions [9]. While the other terminal, reference electrode (RE), silver conductive ink is coated, dried and cleaned with acetone/IPA and DI water and then it is dipped in sodium hypochlorite solution( $\mathrm{NaOCl} 4 \%$ ) to produce a top layer of $\mathrm{AgCl}$ as shown in figure 2. Highly stable and low cost Ag/AgCl electrode with the drift potential of less than $2 \mathrm{mV}$ is used as pseudo RE [8]. The principle mechanism behind our sensing approach is transduction of ionic stimulus into electrical signal. Concentration dependent change in the ISM potential at WE/electrolyte interface prompts a corresponding adjustment in overall gate potential, thus modifying the charge transport in FET. Sensitivity and linearity of potassium ion $\left(\mathrm{K}^{+}\right) \mathrm{WE}$ is determined by potentiometric measurements at several concentrations ranging from $10^{-6} \mathrm{M}$ to $1 \mathrm{M}$. The electrodes are reusable after each measurement by simply rinsing it in deionized water. During fabrication, 15 minute ultrasonication in acetone/IPA/DI water is not sufficient enough for removing organic contamination. Therefore, one more essential step of 30 minute ultrasonication in a solution containing 5:1:1 water, ammonium hydroxide (20\%) and hydrogen peroxide (30\%)is required [10].

(a)

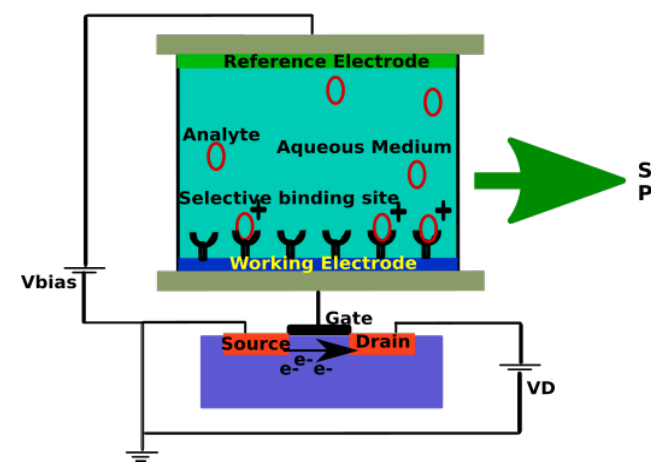

(b)

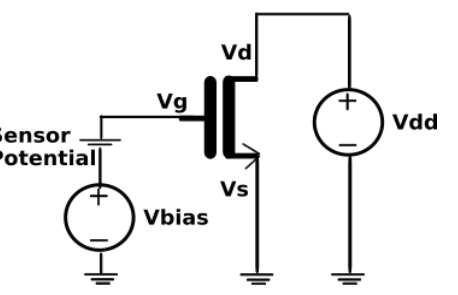

Figure 1: Conceptual illustration of Extended gate FET and experimental setup for $\mathrm{K}^{+}$ detection

\section{Results}

Potentiometric response can be defined by extension form of Nernst equation as follows.

$$
E M F=E^{0}+\frac{2.303 R T}{F z_{i}} \log \left(a_{i}+K_{i, j}^{p o t} a_{j}\right)
$$



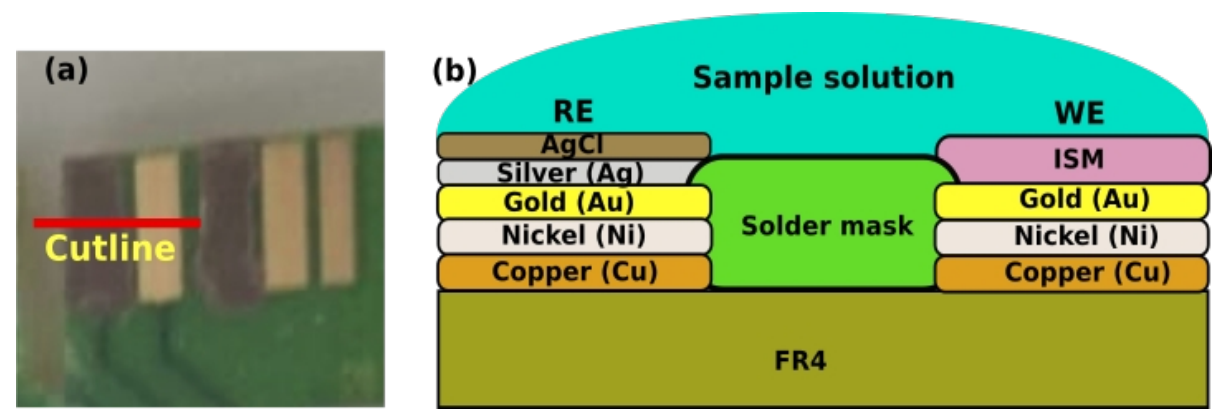

Figure 2: (a) Top view of electrodes on PCB substrate. (b) Cross-sectional schematic view along the cut-line in top view of sensing device on PCB.

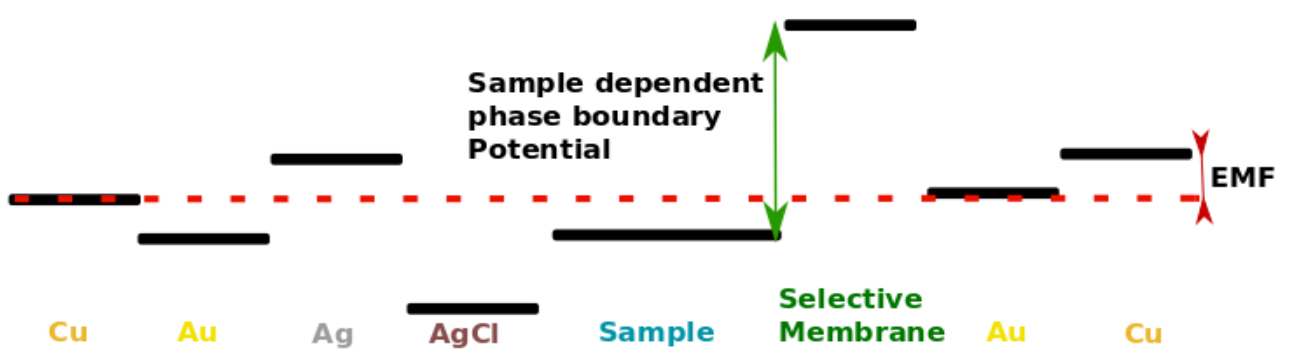

Figure 3: Ideal phase boundary condition for ideal zero current potentiometric measurement (EMF generation between WE and RE)

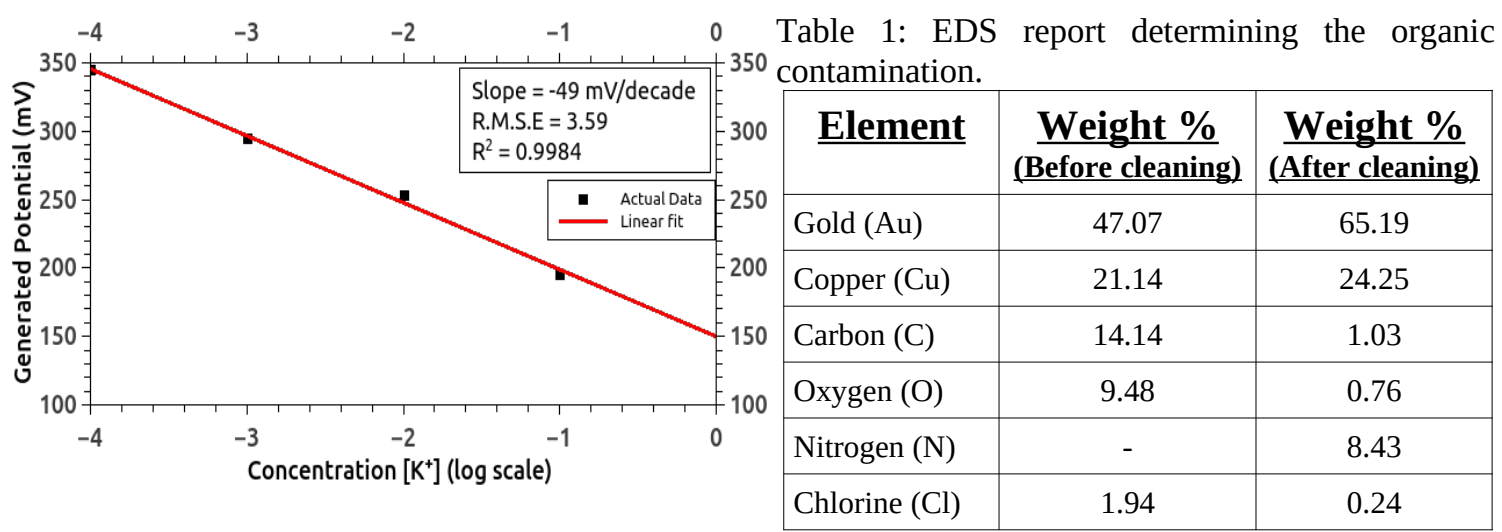

Figure 4: Potentiometric response of device in the linear range

In (1), $a_{i}$ is the concentration of primary ion and $a_{j}$ is the concentration of interfering ion, $\mathrm{R}$ is gas constant, $\mathrm{F}$ is Faraday constant, $\mathrm{T}$ is temperature, and $K_{i, j}^{\text {pot }}$ is selectivity coefficient. $\mathrm{E}^{0}$ is the intercept depend on the work-function of the electrode-electrolyte junction. It represents the difference in free energy of transferring ions from electrolyte to ion selective membrane (ISM) layer. This equation can be simplified to Nernst equation in the absence of interfering ion j. Figure 3 represents the ideal phase boundary of the device fabricated. It has been observed that generated potential across device electrodes exhibits linear response in the range of $10^{-4} \mathrm{M}$ to $1 \mathrm{M}$ with the slope of $49 \mathrm{mV}$ and an intercept at $149 \mathrm{mV}$ (Figure 4). Our study shows that voltage biasing does not affect the electromotive force $\left(\mathrm{V}_{\text {cell }}\right)$. This makes the device suitable and robust for EGFET configuration to enhance the sensitivity for the ion detection applications. Two-fold increase in the sensitivity (100 $\mathrm{mV} /$ decade, much higher than reported values ion selective electrodes $[5,11]$ ) is observed in EGFET configuration. Table 1 represents the 
necessity of organic contamination cleaning before fabrication of working and reference electrode.

\section{Conclusion}

In this study, we presented the label-free and selective detection of potassium ion using ionophore coated ISE. The charge build-up due to helmholtz capacitance across ISE will induce the potential (EMF) across the ionophore interface and hence contributing to the direct determination of potassium ion. Further sensitivity is improved by utilizing induced potential across the gate of FET. Thus, label-free enhanced sensitivity can be achieved using extended gate FET configuration on PBC platform. The innovative approach used in our solid-state $\mathrm{K}^{+}$EGFET has great potential to propel the future in micro-devices with commercialized product for the applications of clinical investigation, environmental and agricultural field. Our methodology represents a handy procedure by using facile fabrication technique for the accomplishment of multipurpose, versatile, and cost-effective ion sensing devices.

\section{References}

1. Dorothee Grieshaber, et al. "Electrochemical Biosensors - Sensor Principles and Architectures”, Sensors, Mar 2008.

2. Jinbo $\mathrm{Hu}$, et al. "Rational design of all-solid-state ion-selective electrodes and reference electrodes”, Trends in Analytical Chemistry 2016.

3. Q. Li et al. "Stable Thin-Film Reference Electrode on Plastic Substrate for All-SolidState Ion-Sensitive Field-Effect Transistor Sensing System," IEEE Electron Device Letters, Oct 2017.

4. T. Goda, et al. "Detection of microenvironmental changes induced by protein adsorption onto self-assembled monolayers using an extended gate-field effect transistor", Anal. Chem., 2011.

5. Salzitsa Anastasova, et al. "Multi-parametric rigid and flexible, low-cost, disposable sensing platforms for biomedical applications”, Biosensors and Bioelectronics, Apr 2018.

6. Prodromakis T, et al. "A novel design approach for developing chemical sensing platform using inexpensive technologies”, IEEE Biomedical Circuits and Systems Conference (BioCAS) 2011.

7. Trantidou $\mathrm{T}$, et al. "The dual role of Parylene $\mathrm{C}$ in chemical sensing: Acting as an encapsulant and as a sensing membrane for $\mathrm{pH}$ monitoring applications” Sens. Actuators B-Chem 2013.

8. Despina Moschou, et al. "Surface and Electrical Characterization of Ag/AgCl Pseudo-Reference Electrodes Manufactured with Commercially Available PCB Technologies”, Sensors, July 2015

9. Sunita Kumbhat, et al. "A potassium-selective electrochemical sensor based on crown-ether functionalized self assembled monolayer”, Journal of Electroanalytical Chemistry, Jan 2018.

10. Gorachand Dutta, et al. "Electrochemical Glucose Biosensor with commercially Fabricated Printed Circuit Board Sensing Electrodes: The importance of Electrode Surface Characteristics in Sensor Sensitivity” IEEE-NEMS 2018.

11. He Q, et al. "Enabling Inkjet Printed Graphene for Ion Selective Electrodes with Postprint Thermal Annealing”, ACS Applied Materials \& Interfaces, Feb 2017. 